\title{
AUTOCUIDADO DO IDOSO DURANTE SUA HOSPITALIZAÇÃO POR INSUFICIÊNCIA CARDÍACA: REVISÃO INTEGRATIVE
}

\section{ARTIGO DE REVISÃO}

GOMES, Lara Alves ${ }^{1}$

ANTONÁCCIO, Renata ${ }^{2}$

ALMEIDA, Geovana Brandão Santana de ${ }^{3}$

FARIA, Luciene Ribeiro de ${ }^{4}$

GOMES, Lara Alves. Et al. Autocuidado do idoso durante sua hospitalização por insuficiência cardíaca: Revisão integrativa. Revista Científica Multidisciplinar Núcleo do Conhecimento. Ano 05, Ed. 12, Vol. 06, pp. 73-84. Dezembro de 2020. ISSN: 2448-0959, Link de acesso: https://www.nucleodoconhecimento.com.br/saude/autocuidado-do-idoso

\section{RESUMO}

A insuficiência cardíaca é um importante fator relacionado ao aumento da dependência funcional, e, quando associada a frequentes internações pode piorar este quadro. Neste contexto, o comportamento de autocuidado associa-se a um menor número de internações por descompensações do quadro clínico, associado a uma melhor qualidade de vida. O objetivo central deste trabalho é compreender como se dá o autocuidado do idoso hospitalizado, além de identificar quais ações de enfermagem contribuem para a manutenção do autocuidado. Trata-se de uma revisão integrativa, que buscou analisar de forma crítica e objetiva, 7 artigos que foram

\footnotetext{
${ }^{1}$ Graduanda em Enfermagem.

2 Doutora em Enfermagem.

${ }^{3}$ Doutora em Enfermagem.

${ }^{4}$ Doutora em Enfermagem.
} 
selecionados por abarcar o autocuidado do idoso no contexto hospitalar. O papel do enfermeiro enquanto educador para estimular o autocuidado foi um fator importante e apontado nas discussões. Além disso, deve ser incorporado no processo de enfermagem planejamento de ações que estimulem o autocuidado do paciente durante sua hospitalização. Conclui-se que existe uma lacuna da literatura com enfoque no autocuidado durante a hospitalização do idoso, além da necessidade de novas pesquisas que incorporem a teoria do autocuidado no contexto da insuficiência cardíaca.

Palavras-chave: Autocuidado, insuficiência cardíaca, idoso, hospitalização, enfermagem.

\section{INTRODUÇÃO}

O autocuidado tal qual concebido pela teorista Dorothea Orem, envolve todas as atividades para a manutenção da vida e seu bem-estar; pressuposto isso, orem fundamenta o autocuidado como uma forma assistencial holística com enfoque predominante na observação e ação sobre a necessidade do outro (COSTA, 2013).

Essa teoria baseia-se fundamentalmente em três pilares: sistema totalmente compensatório, sistema parcialmente compensatório, e sistema de apoio-educação. Nesse ínterim, o sistema apoio-educação é uma das ferramentas principais do enfermeiro para empoderar o paciente, melhorando assim a qualidade assistencial (NICOLATO, 2016).

No contexto das doenças crônicas não transmissíveis, o autocuidado correlata-se à manutenção do bem-estar do usuário em todas as esferas da sua vida (físicas, biopsicossociais, dentre outras), inclusive reduzindo a morbimortalidade, sempre visando autonomia e melhora da qualidade de vida (CONCEIÇÃO et al, 2015).

A insuficiência cardíaca (IC) é uma disfunção com etiologia em alterações funcionais ou estruturais, com sinais e sintomas específicos - sendo os principais: dispneia, ortopneia, dispneia paroxística noturna, fadiga, intolerância ao exercício, tosse 
noturna, ganho de peso, dor abdominal, perda de apetite associada a perda de peso, noctúria, oligúria, edema periférico, hepatomegalia e ascite, extremidades frias e taquicardia - resultantes da alteração no débito cardíaco e/ou elevadas pressões de enchimento em quadros de esforço ou repouso (Sociedade Brasileira de Cardiologia - SBC, 2018).

Essa cardiopatia é a primeira causa de readmissões hospitalares em pacientes idosos - especialmente no primeiro mês após a alta em decorrência de internação por IC aguda. $\mathrm{Na}$ literatura encontramos várias referências que abordam o prognóstico a longo prazo da IC crônica, mas, nota-se uma escassez de estudos voltados aos pacientes descompensados, independentemente se a etiologia for IC crônica ou aguda (DELGADO et al, 2016).

No Brasil, o estudo Brazilian Registry of Acute Heart Failure (BREATHE), demonstrou que a principal causa de reinternações hospitalares é a deficiência na adesão ao tratamento da IC, apontando que o Brasil possui uma das maiores taxas de mortalidade intra-hospitalar em decorrência de IC descompensada. (ALBUQUERQUE et al, 2015).

De acordo com dados epidemiológicos provenientes do DataSUS, nos meses de julho de 2019 a julho de 2020, ocorreram 85.836 internações em decorrência da insuficiência cardíaca apenas na região sudeste do Brasil, resultando em uma média de 7.153 internações ao mês (SIH/SUS, 2020).

Sabendo que a IC é o desfecho final de todas as doenças cardíacas, e sendo um problema de saúde pública devido seus elevados índices de incidência e prevalência associados ao elevado custo ao sistema de saúde, a promoção do autocuidado nesses pacientes vem como um encorajamento para a tomada de decisão, com o objetivo de que o paciente mantenha a estabilidade do seu processo saúde-doença (MESQUITA et al, 2017; PEDRÃO et al, 2018).

No Brasil, o comportamento do autocuidado como um todo é pouco estudado, além disso, fatores individuais que influenciam esse contexto são pouco investigados na 
população com IC (CONCEIÇÃO, 2015), ao passo que no exterior, a educação para o autocuidado é estimulada e realizada por enfermeiros, (ROSS, 2015).

Sabe-se que a hospitalização se associa ao aumento da dependência funcional dos pacientes, corrobora-se ainda que a IC naturalmente é um preditor de dependência funcional em idosos, uma vez que pode afetar todas as suas atividades de vida diárias (XAVIER et al, 2015).

Logo, estudar o autocuidado no contexto da IC com enfoque em idosos hospitalizados, faz-se extremamente necessário - uma vez que há carência desses estudos no Brasil, somado ao índice epidemiológico do nosso país.

\section{OBJETIVOS}

\subsection{OBJETIVO GERAL}

Compreender o autocuidado do idoso hospitalizado.

\subsection{OBJETIVO ESPECÍFICO}

Identificar quais ações de enfermagem contribuem para a manutenção do autocuidado.

\section{METODOLOGIA}

Trata-se de um estudo de revisão integrativa da literatura, com análise de publicações que trazem à luz discussões sobre métodos e os resultados da pesquisa (MENDES, 2008), para discorrer sobre o autocuidado focado no idoso portador de insuficiência cardíaca durante sua hospitalização.

A pesquisa foi realizada nas bases de dados PubMed, BVS, Bireme - BDENF, e Periódicos CAPES. Os descritores utilizados em inglês foram "self care", "aged", "heart failure", "hospitalization", "nursing", com delimitação temporária de publicações entre 2015 e 2020, e o operador booleano AND. 
O critério de inclusão para leitura dos artigos foi incluir no título e no resumo, o autocuidado no ambiente hospitalar em pacientes com insuficiência cardíaca, além de artigos em inglês e em português. Foram excluídos, estudos de revisão e estudos em que a população alvo não fosse composta por idosos.

Tabela 1 - artigos encontrados em cada base de dados

\section{Artigos encontrados em cada base de dados}

\begin{tabular}{|l|l|}
\hline Base de dados & Quantidade de artigos encontrados em cada uma \\
\hline PubMed & 91 artigos \\
\hline BVS & 90 artigos \\
\hline Bireme - BDENF & 24 artigos e 8 teses \\
\hline Periódicos CAPES & 7 artigos \\
\hline
\end{tabular}

Fonte: Própria autora, 2020.

$\mathrm{Na}$ pesquisa inicial, foram encontrados 230 artigos, estando associados aos descritores. Após a leitura do título e do resumo, foram selecionados 38 artigos para análise, sendo que cada artigo foi contabilizado apenas uma vez. Depois de uma leitura do artigo como um todo, foram identificados apenas 7 artigos elegíveis para análise.

\section{RESULTADOS E DISCUSSÃO}

Após a leitura dos artigos, foi criado e preenchido um instrumento que abarca autores e o ano de publicação, objetivos e metodologia.

Tabela 2 - Publicações analisadas:

\begin{tabular}{|l|l|l|l|l|}
\hline Estudo & $\begin{array}{l}\text { Autores e } \\
\text { ano de } \\
\text { publicação }\end{array}$ & $\begin{array}{l}\text { Principais ações } \\
\text { de enfermagem }\end{array}$ & Metodologia \\
\hline
\end{tabular}




\begin{tabular}{|c|c|c|c|c|}
\hline R1 & $\begin{array}{l}\text { Reeder, K. } \\
\text { M., Ercole, } \\
\text { P. M., Peek, } \\
\text { G. M., \& } \\
\text { Smith, C. E. } \\
\text { (2015). }\end{array}$ & $\begin{array}{l}\text { Explorar as } \\
\text { percepções dos } \\
\text { pacientes sobre os } \\
\text { sintomas } \\
\text { comportamentos de } \\
\text { autocuidado para o } \\
\text { alívio dos sintomas, e } \\
\text { o tempo que levou à } \\
\text { hospitalização por } \\
\text { insuficiência } \\
\text { cardíaca. }\end{array}$ & $\begin{array}{l}\text { Neste artigo, } \\
\text { vemos que a } \\
\text { educação e o grau } \\
\text { de instrução } \\
\text { afetam } \\
\text { autocuidado; } \\
\text { porém, não traz } \\
\text { ações específicas } \\
\text { da equipe de } \\
\text { enfermagem. }\end{array}$ & $\begin{array}{l}\text { Estudo } \\
\text { transversal } \\
\text { exploratório }\end{array}$ \\
\hline R2 & 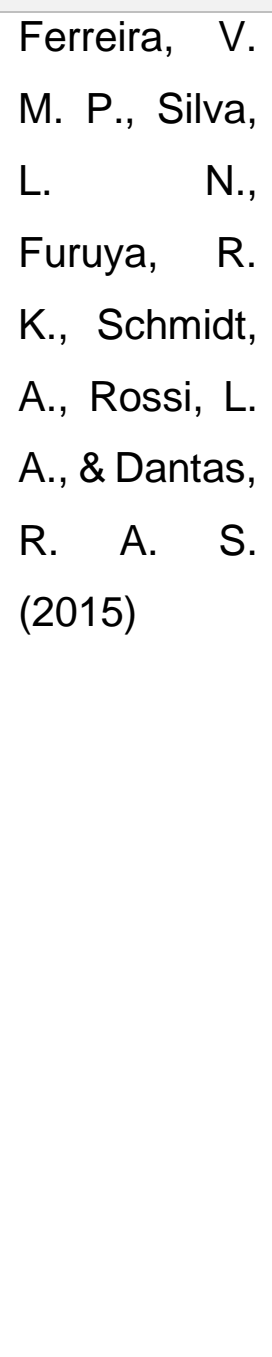 & $\begin{array}{l}\text { Avaliar os } \\
\text { comportamentos de } \\
\text { autocuidado em } \\
\text { pacientes } \\
\text { hospitalizados com } \\
\text { insuficiência cardíaca } \\
\text { descompensada, } \\
\text { considerando sexo, } \\
\text { sintomas depressivos } \\
\text { e senso de coerência; } \\
\text { além de comparar as } \\
\text { medidas de } \\
\text { depressão e senso } \\
\text { de coerência } \\
\text { segundo o sexo dos } \\
\text { pacientes } \\
\text { hospitalizados por } \\
\text { insuficiência cardíaca } \\
\text { descompensada }\end{array}$ & $\begin{array}{l}\text { O planejamento } \\
\text { da assistência de } \\
\text { enfermagem deve } \\
\text { ser feito com o } \\
\text { objetivo de } \\
\text { promover o } \\
\text { autocuidado, além } \\
\text { de medidas } \\
\text { educacionais } \\
\text { visando } \\
\text { conhecimento da } \\
\text { doença. }\end{array}$ & $\begin{array}{l}\text { Estudo } \\
\text { correlacional } \\
\text { de corte } \\
\text { transversal }\end{array}$ \\
\hline
\end{tabular}




\begin{tabular}{|c|c|c|c|c|}
\hline R3 & $\begin{array}{l}\text { Linn, A. C., } \\
\text { Azzolin, K., } \\
\text { \& Souza, E. } \\
\text { N. D. (2016) }\end{array}$ & 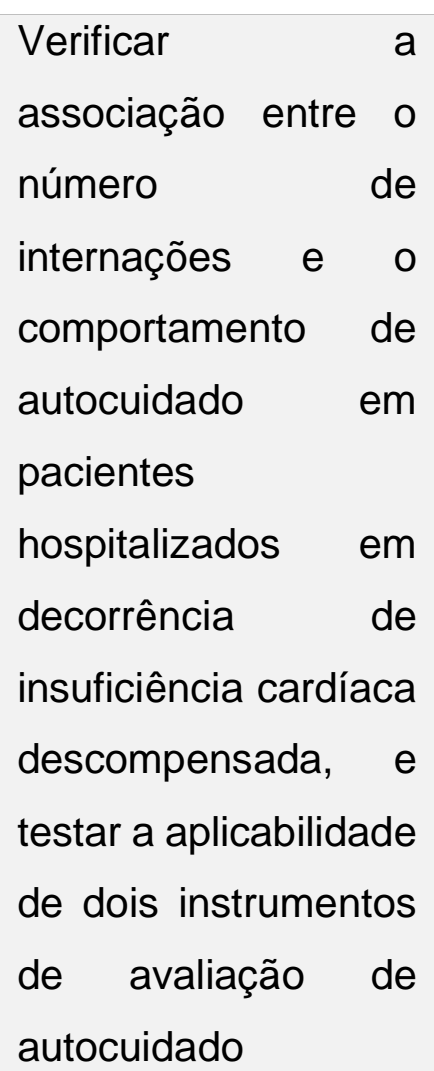 & $\begin{array}{l}\text { Educação em } \\
\text { saúde por parte } \\
\text { da equipe de } \\
\text { enfermagem é } \\
\text { uma importante } \\
\text { ação com } \\
\text { resultados } \\
\text { expressivos. Além } \\
\text { disso, a prática } \\
\text { assistencial deve } \\
\text { ser holística, } \\
\text { levando em conta } \\
\text { o biopsicossocial } \\
\text { do indivíduo. }\end{array}$ & $\begin{array}{l}\text { Longitudinal } \\
\text { retrospectivo, } \\
\text { quantitativo }\end{array}$ \\
\hline R4 & $\begin{array}{l}\text { Sposito, N. } \\
\text { P. B., \& } \\
\text { Kobayashi, } \\
\text { R. M. (2016). }\end{array}$ & $\begin{array}{l}\text { Caracterizar o perfil } \\
\text { das mulheres adultas } \\
\text { internadas em } \\
\text { enfermarias de um } \\
\text { hospital público } \\
\text { cardiológico } \\
\text { localizado na cidade } \\
\text { de São Paulo (Brasil), } \\
\text { que tinham como } \\
\text { diagnóstico médico } \\
\text { de internação } \\
\text { a insuficiência } \\
\text { cardíaca; identificar o } \\
\text { conhecimento } \\
\text { dessas pacientes } \\
\text { sobre a doença e seu }\end{array}$ & 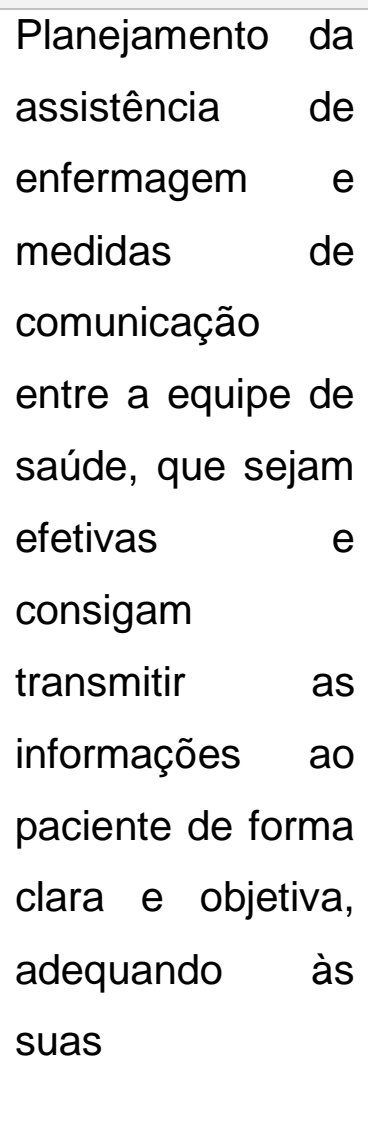 & $\begin{array}{l}\text { Exploratório e } \\
\text { descritivo }\end{array}$ \\
\hline
\end{tabular}




\begin{tabular}{|c|c|c|c|c|}
\hline & & $\begin{array}{lr}\text { tratamento; } & \text { e } \\
\text { descrever } & \text { os } \\
\text { diagnósticos } & \text { de } \\
\text { enfermagem e as } \\
\text { prescrições } \\
\text { enfermagem } \\
\text { relacionadas } \\
\text { à insuficiência } \\
\text { cardíaca durante sua } \\
\text { internação }\end{array}$ & $\begin{array}{l}\text { necessidades } \\
\text { educacionais. }\end{array}$ & \\
\hline R5 & $\begin{array}{l}\text { Medeiros, J., } \\
\text { \& Medeiros, } \\
\text { C. D. A. } \\
(2017) .\end{array}$ & $\begin{array}{l}\text { Caracterizar o perfil } \\
\text { clínico } \\
\text { sociodemográfico } \\
\text { dos portadores de } \\
\text { insuficiência cardíaca } \\
\text { e descrever o } \\
\text { comportamento de } \\
\text { autocuidado de } \\
\text { pacientes adultos } \\
\text { com insuficiência } \\
\text { cardíaca, por meio da } \\
\text { escala Self-Care of } \\
\text { Heart Failure Index - } \\
\text { SCHFI, versão } \\
\text { brasileira. }\end{array}$ & $\begin{array}{l}\text { Principais ações } \\
\text { de enfermagem } \\
\text { apontadas neste } \\
\text { artigo foram de } \\
\text { cunho educativo } \\
\text { (educação em } \\
\text { saúde direcionada } \\
\text { ao autocuidado) e } \\
\text { assistencial } \\
\text { (planejamento } \\
\text { assistencial a fim } \\
\text { de abarcar } \\
\text { hospitalização e } \\
\text { alta. }\end{array}$ & $\begin{array}{l}\text { Estudo } \\
\text { observacional } \\
\text { transversal }\end{array}$ \\
\hline R6 & $\begin{array}{l}\text { Mlynarska, } \\
\text { A., Golba, K. } \\
\text { S., \& } \\
\text { Mlynarski, R. } \\
(2018)\end{array}$ & $\begin{array}{l}\text { Avaliar o efeito do } \\
\text { método terapêutico } \\
\text { da insuficiência } \\
\text { cardíaca, o grau de } \\
\text { aceitação da doença } \\
\text { e a ocorrência da }\end{array}$ & $\begin{array}{lr}\text { Ações voltadas à } \\
\text { educação } & \text { em } \\
\text { saúde } & \text { com } \\
\text { enfoque } & \text { na } \\
\text { abordagem não } & \text { farmacológica do }\end{array}$ & $\begin{array}{l}\text { Estudo } \\
\text { transversal } \\
\text { quantitativo }\end{array}$ \\
\hline
\end{tabular}




\begin{tabular}{|c|c|c|c|c|}
\hline & & $\begin{array}{l}\text { síndrome da } \\
\text { fragilidade na adesão } \\
\text { às recomendações } \\
\text { terapêuticas e } \\
\text { autocuidado no } \\
\text { pacientes em } \\
\text { insuficiência } \\
\text { cardíaca. }\end{array}$ & $\begin{array}{l}\text { tratamento da } \\
\text { insuficiência } \\
\text { cardíaca. }\end{array}$ & \\
\hline R7 & $\begin{array}{l}\text { Born, M. C., } \\
\text { Azzolin, K. } \\
\text { D. O., \& } \\
\text { Souza, E. N. } \\
\text { D. (2019) }\end{array}$ & $\begin{array}{l}\text { Identificar os sinais e } \\
\text { sintomas de } \\
\text { descompensação da } \\
\text { insuficiência cardíaca } \\
\text { e o tempo de duração } \\
\text { até a admissão na } \\
\text { emergência } \\
\text { hospitalar. }\end{array}$ & $\begin{array}{l}\text { As ações de } \\
\text { enfermagem } \\
\text { apontadas foram } \\
\text { de cunho } \\
\text { educativo, } \\
\text { direcionadas a } \\
\text { identificação dos } \\
\text { sinais e sintomas } \\
\text { de piora e melhora } \\
\text { do seu estado de } \\
\text { saúde. }\end{array}$ & $\begin{array}{l}\text { Estudo } \\
\text { transversal } \\
\text { quantitativo }\end{array}$ \\
\hline
\end{tabular}

Fonte: própria autora, 2020.

Apesar da carência em referenciais teóricos no Brasil, a maioria dos artigos (R2, R3, R4, R5 e R7) são nacionais; um (R1) foi publicado nos EUA e um (R6) publicado na Polônia. O comportamento do autocuidado no ambiente intra-hospitalar é um aspecto pouco estudado como demonstrado pelo número baixo de publicações encontradas que dissertam sobre o assunto. A maioria dos artigos encontrados antes da préanálise, foram pesquisas realizadas em ambiente ambulatorial ou em um contexto comparativo - durante a internação e após a alta, o que não era o foco da pesquisa, o que reforça a percepção de que há uma lacuna bibliográfica em estudos com enfoque exclusivo durante a hospitalização. 
O perfil sociodemográfico foi bastante variado: apenas um estudo estipulou como prérequisito pacientes acima de 60 anos (R6), os demais, realizaram entrevistas e analisaram todos os pacientes hospitalizados durante o período de coleta de dados, gerando uma variância de idade maior; apesar disso, a maioria dos hospitalizados estava na faixa etária acima de 60 anos, o que permitiu que os estudos fossem analisados. Apenas um estudo (R4) focou sua coleta de dados em mulheres, os demais entrevistaram ambos os sexos.

Todos os artigos analisados chegaram à conclusão de que o conhecimento sobre a doença afeta o autocuidado, e, que quanto maior a escolaridade, melhor é o índice de autocuidado. Muitos pacientes não têm conhecimento sobre a doença nem sobre os fatores de exacerbação e piora da insuficiência cardíaca (R1, R2, R3 R4, R5, R6 e $\mathrm{R} 7)$.

Outro ponto amplamente citado entre os autores, são as ações de enfermagem que auxiliam na manutenção do autocuidado ( $R 2, R 3, R 4, R 5$ e R7). O planejamento da assistência visando a promoção do autocuidado contribui para melhorar o estado de saúde e foi apontado como importante estratégia para os enfermeiros durante a prestação da assistência (R2).

O planejamento da assistência de enfermagem atesta o compromisso junto ao paciente, haja visto os diagnósticos e prescrições dos cuidados de enfermagem. No contexto da hospitalização, é fundamental que o planejamento contemple a alta hospitalar e as orientações que vão ser repassadas ao paciente naquele momento. Para que suas carências sejam supridas, as ações assistenciais devem estar de acordo com sua visão biopsicossocial (CARNEIRO et al, 2020).

Além disso, ações educativas foram constantemente apontadas e frisadas pelos autores como importante estratégias na promoção do autocuidado (R3, R5 e R7), mostrando o relevante papel na educação em saúde que cabe aos enfermeiros - em especial na atenção especializada. As ações educativas atuam como ferramentas imprescindíveis na promoção do autocuidado, em especial se tratando da saúde do 
idoso, uma vez que ampliam sua independência, além de melhorar a sua qualidade de vida (DE LIMA et al, 2017).

A comunicação efetiva entre equipe de saúde e paciente também foi um aspecto assistencial que merece atenção (R4). Quando tratamos de abordagens metodológicas educacionais, a comunicação deve se adequar ao paciente em todos os aspectos: sua idade, cultura, grau de instrução, devendo o profissional estar atento em como o cliente está absorvendo a informação repassada, uma vez que para o seu empoderamento e autocuidado, as orientações devem e necessitam ser absorvidas (BRAGA et al, 2020).

No tocante ao binômio paciente e enfermeiro, é impreterível o acolhimento e orientação direcionadas à situação e demanda, ao passo que promovemos o acesso à educação em saúde de forma eficaz, é notório uma adaptação do paciente às dificuldades além de uma melhora no seu índice de autocuidado (FARIAS 2019).

Apesar dos artigos não destacarem o autocuidado durante a hospitalização, foi avaliado o autocuidado dos pacientes na maioria destes (R2, R3, R5 e R6). Em pacientes com insuficiência cardíaca, a manutenção do autocuidado em especial, o conhecimento da sua -doença bem como suas descompensações, está relacionado com redução do número de readmissões hospitalares em um curto período de tempo, fomentando a tese de que a educação em saúde no âmbito hospitalar focada no autocuidado possui resultados positivos (AWOKE et al, 2019).

\section{CONSIDERAÇÕES FINAIS}

Devido aos índices crescentes de diagnósticos de insuficiência cardíaca atrelados ao envelhecimento populacional, pesquisas relacionadas a tal temática são relevantes. O enfermeiro tem um papel fundamental neste contexto, uma vez que suas atividades assistenciais e educativas voltadas a este público, podem reduzir o índice de internações além de proporcionar melhora do estado de saúde. 
Dentre os objetivos traçados, nos deparamos com dificuldade em encontrar artigos que avaliassem o autocuidado dos idosos durante a sua internação; os artigos encontrados que realizaram a avaliação do autocuidado por meio de escalas, não avaliaram apenas sua hospitalização. Além disso, há uma carência em publicações que explorem como a internação afeta 0 autocuidado do idoso com base nas suas percepções individuais, levando em conta que no ambiente hospitalar estes estão expostos a cuidados integrais por meio da equipe de enfermagem.

A metodologia de pesquisa foi adequada para a busca e análise de artigos que trouxessem o autocuidado de idosos durante sua internação, como o foco principal. A análise dos artigos encontrados foi feita de forma criteriosa, sempre buscando um ponto em comum entre eles, além de buscar diferenças e aspectos únicos, mas que respondem o questionamento principal: como se dá o autocuidado do idoso portador de insuficiência cardíaca.

Os resultados foram ao encontro do que atualmente encontra-se publicado na literatura, em especial no que tange o papel do enfermeiro enquanto educador de saúde. Além disso, muitos artigos não foram incluídos na análise por se tratar de pesquisas comparativas - durante a hospitalização e no acompanhamento pós-alta, ressaltando a carência de estudos apenas sob a ótica hospitalar. Apenas um estudo analisado focou nos idosos, os demais incluíram todos os indivíduos hospitalizados apesar da maioria participante ser idosa, esta foi uma limitação encontrada durante a coleta e análise dos dados.

Um importante resultado encontrado, foi sobre como a comunicação entre a equipe de saúde afeta as percepções do paciente sobre sua saúde, apontando para possibilidades posteriores de estudos e pesquisas, no contexto do idoso portador de insuficiência cardíaca. Ressalta-se que o profissional deve estar atento em como repassar as informações para o paciente, sempre se adequando às suas necessidades, para que o objetivo da educação para o autocuidado seja atingido. 
Recomenda-se que novas pesquisas sejam realizadas para avaliar o autocuidado durante a hospitalização em portadores de insuficiência cardíaca, a fim de contribuir para ampliar o conhecimento do tema proposto.

\section{REFERÊNCIAS}

ALBUQUERQUE, Denilson Campos de; NETO, João David de Souza; BACAL, Fernando; ROHDE, Luiz Eduardo Paim; BERNARDEZ-PEREIRA, Sabrina; BERWANGER, Otavio; ALMEIDA, Dirceu Rodrigues. I Brazilian registry of heart failure (BREATHE) - clinical aspects, care quality and hospitalization outcomes. Arquivos Brasileiros de Cardiologia. 2015. p. 433-442.

AWOKE, Martha S.; BAPTISTE, Diana-Lyn; DAVIDSON, Patricia; ROBERTS, Allen; DENNISON-HIMMELFARB, Cheryl. A quasi-experimental study examining a nurse-led education program to improve knowledge, self-care, and reduce readmission for individuals with heart failure. Contemporary Nurse, [S.L.], v. 55, n. 1, p. 15-26, 2 jan. 2019. Informa UK Limited. http://dx.doi.org/10.1080/10376178.2019.1568198.

BRAGA, Bruna Rodrigues, et al. Enfermagem e clientes hospitalizados: a comunicação em uma unidade militar. Rev. enferm. UFPE on line, 2020, [1-9].

BORN, Maraísa Carine; AZZOLIN, Karina de Oliveira; SOUZA, Emiliane Nogueira de. How long before hospital admission do the symptoms of heart failure decompensation arise? Revista Latino-Americana de Enfermagem, [S.L.], v. 27, p. 1-8, 2019. FapUNIFESP (SciELO). http://dx.doi.org/10.1590/1518-8345.2735.3119.

CARNEIRO, Jayanne Moreira; JESUS, Larissa Oliveira de; SILVA, Caroline Santos; SANTIAGO, Ainara dos Santos; SANTOS, Anderson Alves Lima; MARQUES, Patrícia Figueiredo. Nursing discharge plan in hospitals: an experience report. Revista de Pesquisa Cuidado É Fundamental Online, [S.L.], p. 1045-1049, 14 ago. 2020. Universidade Federal do Estado do Rio de Janeiro UNIRIO. http://dx.doi.org/10.9789/2175-5361.rpcfo.v12.7495. 
CONCEIÇÃO, Ana Paula da; SANTOS, Mariana Alvina dos; SANTOS, Bernardo dos; CRUZ, Diná de Almeida Lopes Monteiro da. Autocuidado de pacientes com insuficiência cardíaca. Revista Latino-Americana de Enfermagem. 2015. p. 578-586.

COSTA, Sibely Rabaca Dias da; CASTRO, Edna Aparecida Barbosa de; ACIOLI Sonia. Capacidade de autocuidado de adultos e idosos hospitalizados: implicações para o cuidado de enfermagem. Revista Mineira de Enfermagem. 2013. p. 192-199.

DE LIMA, Paula Alves, et al. Educational activities on cardiovascular health for the elderly people at home. Journal of Nursing UFPE on line, 2017. 11.11: 44984504.

DELGADO, Anne et al. Registro de Insuficiência Cardíaca Aguda: Modelo de Avaliação de Risco na Insuficiência Cardíaca Descompensada. Arq. Bras. Cardiol., São Paulo , v. 107, n. 6, p. 557-567, Dec. 2016 . Available from $<$ http://www.scielo.br/scielo.php?script=sci_arttext\&pid=S0066782X2016004500557\&Ing=en\&nrm=iso > . access on 27 Sept. 2020.

FARIAS, Dilton Luis Soares de; NERY, Roberta Nayara Barroso; SANTANA, Mary Elizabeth de. $O$ enfermeiro como educador em saúde da pessoa estomizada com câncer colorretal. Enferm. foco (Brasília), 2019, 35-39.

FERREIRA, Viviane Martinelli Pelegrino; SILVA, Luma Nascimento; FURUYA, Rejane Kiyomi; SCHMIDT, André; ROSSI, Lídia Aparecida; DANTAS, Rosana Aparecida Spadoti. Autocuidado, senso de coerência e depressão em pacientes hospitalizados por insuficiência cardíaca descompensada. Revista da Escola de Enfermagem da USP. 2015. p. 388-394.

LINN, Amanda Chlalup; AZZOLIN, Karina; SOUZA, Emiliane Nogueira de. Associação entre autocuidado e reinternação hospitalar de pacientes com insuficiência cardíaca. Revista Brasileira de Enfermagem, [S.L.], v. 69, n. 3, p. 500506, jun. 2016. FapUNIFESP (SciELO). http://dx.doi.org/10.1590/0034$7167.2016690312 \mathrm{i}$. 
MEDEIROS, Juliana; MEDEIROS, Carolina de Araújo. Avaliação do autocuidado nos portadores de insuficiência cardíaca. Cogitare Enfermagem. Universidade Federal do Paraná. 2017. p. 1-10.

MESQUITA, Evandro Tinoco, et al. Entendendo a hospitalização em pacientes com insuficiência cardíaca. International Journal of Cardiovascular Sciences, 2017, 30.1: 81-90.

NICOLATO, Fernanda Vieira; COUTO, Alcimar Marcelo do; CASTRO, Edna Aparecida Barbosa de. Capacidade de autocuidado de idosos atendidos pela consulta de enfermagem na atenção secundária à saúde. Revista de enfermagem do centro-oeste mineiro. 2016. p. 2199-2211.

PEDRÃO, Thais Gassi; BRUNORI, Evelise Helena Fadini Reis; SANTOS, Eloiza da Silva; BEZERRA, Amanda; SIMONETTI, Sérgio Henrique. Diagnósticos e intervenções de enfermagem para pacientes cardiológicos em cuidados paliativos. Revista de Enfermagem Ufpe On Line, [S.L.], v. 12, n. 11, p. 3038-3045, 6 nov. 2018. Revista de Enfermagem, UFPE Online. http://dx.doi.org/10.5205/19818963-v12i11a234933p3038-3045-2018.

REEDER, Katherine M., et al. Symptom perceptions and self-care behaviors in patients who self-manage heart failure. The Journal of cardiovascular nursing, 2015, 30.1: E1.

ROSS, Anita; OHLSSON, Ulla; BLOMBERG, Karin; GUSTAFSSON, Margareta. Evaluation of an intervention to individualise patient education at a nurse-led heart failure clinic: a mixed-method study. Journal Of Clinical Nursing, [s.I.], v. 24, n. 11-12, p.1594-1602, 5 mar. 2015.

SIH/SUS, Ministério da Saúde - Sistema de Informações Hospitalares do SUS. Morbidade hospitalar do sus: por local de internação - Brasil. 2020: Insuficiência cardíaca na região sudeste. Disponível em: http://tabnet.datasus.gov.br/cgi/tabcgi.exe?sih/cnv/niuf.def. Acesso em: 27 set. 2020. 
SOCIEDADE BRASILEIRA DE CARDIOLOGIA (SBC). Diretriz Brasileira de Insuficiência Cardíaca Crônica e Aguda. Arquivos Brasileiros de Cardiologia. 2018. p. 436-539.

SPOSITO, Natália Pinheiro Braga; KOBAYASHI, Rika Miyahara. Knowledge of women with heart failure. Reme: Revista Mineira de Enfermagem, [S.L.], v. 20, p. 17, 2016. GN1 Genesis Network. http://dx.doi.org/10.5935/1415-2762.20160052.

XAVIER, Sara de Oliveira; FERRETTI-REBUSTINI, Renata Eloah de Lucena; SANTANA-SANTOS, Eduesley; LUCCHESI, Paola Alves de Oliveira; HOHL, Karine Generoso. Insuficiência cardíaca como preditor de dependência funcional em idosos hospitalizados. Revista da Escola de Enfermagem da USP. 2015. p. 790796.

Enviado: Novembro, 2020.

Aprovado: Dezembro, 2020. 